\title{
Immaturity of Visual Fixations in Dyslexic Children
}

\author{
Aimé Tiadi ${ }^{1 *}$, Christophe-Loïc Gérard ${ }^{2}$, Hugo Peyre ${ }^{2}$, Emmanuel Bui-Quoc ${ }^{3}$ \\ and Maria Pia Bucci ${ }^{1}$ \\ ${ }^{1}$ UMR 1141, Institut National de la Santé et de la Recherche Médicale - Université Paris Diderot - Paris 7, Robert Debré \\ Hospital, Paris, France, ${ }^{2}$ Child and Adolescent Psychiatry Department, Robert Debré Hospital, Paris, France, \\ ${ }^{3}$ Ophthalmology Department, Robert Debré Hospital, Paris, France
}

OPEN ACCESS

Edited by:

Arthur M. Jacobs,

Freie Universität Berlin, Germany

Reviewed by:

Jascha Ruesseler,

University of Bamberg, Germany

Stefan Hawelka,

University of Salzburg, Austria

${ }^{*}$ Correspondence:

Aimé Tiadi

bi-kuyami-guy-aime.tiadi@inserm.fr

Received: 03 November 2015 Accepted: 05 February 2016 Published: 17 February 2016

Citation:

Tiadi A, Gérard C-L, Peyre H, Bui-Quoc E and Bucci MP (2016) Immaturity of Visual Fixations in Dyslexic Children.

Front. Hum. Neurosci. 10:58. doi: 10.3389/fnhum.2016.00058
To our knowledge, behavioral studies recording visual fixations abilities in dyslexic children are scarce. The object of this article is to explore further the visual fixation ability in dyslexics compared to chronological age-matched and reading age-matched non-dyslexic children. Fifty-five dyslexic children from 7 to 14 years old, 55 chronological age-matched non-dyslexic children and 55 reading age-matched non-dyslexic children participated to this study. Eye movements from both eyes were recorded horizontally and vertically by a video-oculography system (EyeBrain ${ }^{\circledR}$ T2). The fixation task consisted in fixating a white-filled circle appearing in the center of the screen for $30 \mathrm{~s}$. Results showed that dyslexic children produced a significantly higher number of unwanted saccades than both groups of non-dyslexic children. Moreover, the number of unwanted saccades significantly decreased with age in both groups of non-dyslexic children, but not in dyslexics. Furthermore, dyslexics made more saccades during the last $15 \mathrm{~s}$ of fixation period with respect to both groups of non-dyslexic children. Such poor visual fixation capability in dyslexic children could be due to impaired attention abilities, as well as to an immaturity of the cortical areas controlling the fixation system.

Keywords: visual fixation, frontal eye field (FEF), superior colliculus (SC), immaturity, dyslexia, children, eye movements, attention

\section{INTRODUCTION}

Visual fixation consists in maintaining an image on the fovea in order to perceive it. Palvidis (1981) was the first to report poor fixation capabilities in 12 dyslexic children with respect to 12 non dyslexic children. More recently, De Luca et al. (1999) explored fixation capability in 10 dyslexic children and in 11 non-dyslexic children of comparable chronological age and they did not find any difference between the two groups of children. These authors suggested that oculomotor differences between dyslexic and non-dyslexic children occurred during reading task only. In the present study, we explored further fixation capability in a large number of dyslexic children and we compared their results with those of two groups of non-dyslexic children having similar chronological and reading age.

Munoz and Wurtz (1992) showed in monkeys that neural cells located in the rostral cells are activated to avoid unwanted saccades. These authors recorded fixation activity and saccade suppression in two monkeys $48 \mathrm{~h}$ after muscimol injection in the rostral cells of the superior colliculus (SC), and showed that muscimol inhibits the neural transmission of the rostral cells. Indeed, after injection of muscimol, the monkeys had more 
difficulty suppressing saccade initiation and several unwanted saccades were initiated before the target switched on. This study showed that rostral cells of the SC are involved in both visual fixation and saccade suppression. In 1995, the same authors explored further the role of SC cells in monkeys and they confirmed that some cells in the rostral pole are responsible for visual fixation while the other cells are responsible for preparation and generation of saccades (Munoz and Wurtz, 1995).

The frontal eye field (FEF) is known to play also an important role in the control of saccades and in their suppression (Leigh and Zee, 2006). For instance, Burman and Bruce (1997) studied suppression of saccades using an electrical micro-stimulation in monkeys eliciting several types of saccades (visual-guided, memory, pro- and anti-saccades). These authors reported that the ventral-lateral area of the FEF, near the arcuate spur, contains a specific and delimited region responsible for saccade suppression. Furthermore, they confirmed previous findings from Segraves and Park (1993) suggesting that the control and inhibition of the saccades is done by the relationship between FEF cells, SC and pontineomnipause region.

Hasegawa et al. (2004) recorded the activity of the neurons in the prefrontal cortex of monkeys in order to explore how this area is involved in the suppression of unwanted saccades. They used an oculomotor task in which monkeys had to remember a stimulus location, i.e., to avoid making a saccade, and they observed a group of neurons in both the FEF and the caudal prefrontal cortex that were activated during saccade suppression.

In patients with frontal lesions, Guitton et al. (1985) reported more unwanted saccades than among control subjects, suggesting that frontal lobes contribute to the suppression of inappropriate saccades. Moreover, Braun et al. (1992) recorded saccades in adult patients with frontal and parietal lesions vs. control subjects, and they showed that patients had greater difficulties suppressing saccades, suggesting a deficit in the fixation control system.

Behavioral studies recording fixation capabilities in children are, to our knowledge, scarce. Biscaldi et al. (1996) compared oculomotor capability in dyslexic and non-dyslexic children and adults (from 12 to 32 years old) and they showed that dyslexics had more express saccades than non-dyslexic subjects, suggesting a poor fixation control among dyslexic subjects. These authors advanced the hypothesis that the deficit in attentional process might be linked with the parietal cortical areas in the dyslexic population. Recently, our group explored the quality of fixation during a dual postural/oculomotor task in both healthy (Ajrezo et al., 2013) and dyslexic children (Bucci et al., 2014). Ajrezo et al. (2013) reported that the quality of fixation during a dual task improved with the increasing age of the children; the number of saccades during fixation was significantly reduced in adolescents, suggesting that the fixation performance in children is underdeveloped, due to the lack of maturation of their cortical areas (Luna and Sweeney, 2001). In line with this thinking, Bucci et al. (2014) studied the quality of fixation during a dual task in 30 dyslexics (from 7 to 13 years old) compared with 30 chronological age-matched and 30 reading age-matched non-dyslexic children. The authors showed poor quality of the fixation in dyslexic children compared to both non-dyslexic groups, which could be in relation with visual attention deficits reported in dyslexic children (Facoetti et al., 2003).

Based on all these findings, the goal of the present study was to further explore visual fixation capability in dyslexic children and to compare these results to those from two groups of chronological and reading age-matched non-dyslexic children. This was done in order to explore further whether fixation instability in dyslexic children was due to the consequence of a reduced reading exposure or rather due to immaturity of cortical areas involved in the visual fixation control.

\section{MATERIALS AND METHODS}

\section{Subjects}

Fifty-five dyslexic children from 7 to 14 years old (mean age: $10.1 \pm 0.2$ years) participated to the study. Dyslexic children were recruited from Robert Debré Pediatric Hospital, to which they had been referred for a complete evaluation of their dyslexia including an extensive examination of their neurological/psychological and phonological capabilities. For each child, we measured the time they required to read a text passage, assessed their general text comprehension, and evaluated their ability to read words and pseudo-words using the L2MA battery (Chevrie-Muller et al., 1997). This is the standard test in France. It was developed by the "Centre de Psychologie Appliquée de Paris" and has been used previously by our team (Bucci et al., 2012, 2013a,b, 2014; Tiadi et al., 2014) to select dyslexic populations. Inclusion criteria were scored on the L2MA which were more than two standard deviations from the mean, and a normal mean intelligence quotient (IQ, evaluated using the WISC-IV), namely between 85 and 115. Any hyperactivity deficit has been excluded by using the ADHD Rating Scale-parental report (ADHD$\mathrm{RS})$.

Fifty-five chronological and reading age-matched non-dyslexic children respectively of $10.4 \pm 0.3$ years old and $8.14 \pm 0.20$ years were also examined. The inclusion criteria were as follows: no known neurological or psychiatric abnormalities, no history of reading difficulty, no visual impairment, or difficulty with near vision. Also, IQ in controls was estimated on two subtests, one assessing the verbal capability (similarities test) and one assessing the logic capability (matrix reasoning test). Normal range for both tests is $10 \pm 3$ (Wechsler intelligence scale for children-fourth edition, 2004). All the healthy children we tested had normal verbal $(10.36 \pm 0.8)$ and logic capabilities $(10.64 \pm 0.5)$. The reading age-matched of all children were assessed by using the ELFE test (cogni-sciences, Grenoble) and Table 1 shows the reading age for each dyslexic children tested.

The investigation adhered to the principles of the Declaration of Helsinki and was approved by our Institutional Human Experimentation Committee. Written consent was obtained 
TABLE 1 | Chronological and reading age of each dyslexic child tested.

\begin{tabular}{lccccc}
\hline Dyslexics & $\begin{array}{c}\text { Chronological } \\
\text { age } \\
\text { SE } \pm \text { 0.2 }\end{array}$ & $\begin{array}{c}\text { Reading } \\
\text { age } \\
\text { SE } \pm \mathbf{0 . 2}\end{array}$ & $\begin{array}{c}\text { Dyslexic } \\
\text { Chronological } \\
\text { age } \\
\text { SE } \pm \mathbf{0 . 2}\end{array}$ & $\begin{array}{c}\text { Reading } \\
\text { age } \\
\text { SE } \pm \mathbf{0 . 2}\end{array}$ \\
\hline D1 & 7.42 & 6 & D29 & 9.30 & 7.5 \\
D2 & 7.60 & 6 & D30 & 9.70 & 7.5 \\
D3 & 7.70 & 6 & D31 & 10.25 & 8 \\
D4 & 7.70 & 6 & D32 & 10.83 & 8 \\
D5 & 7.75 & 6 & D33 & 10.10 & 8 \\
D6 & 7.80 & 6 & D34 & 10.80 & 8 \\
D7 & 7.92 & 6 & D35 & 10.30 & 8 \\
D8 & 8.00 & 6 & D36 & 10.50 & 8 \\
D9 & 8.10 & 6 & D37 & 11.42 & 9 \\
D10 & 8.20 & 6 & D38 & 11.58 & 9 \\
D11 & 8.20 & 6 & D39 & 11.42 & 9 \\
D12 & 8.25 & 7 & D40 & 11.50 & 9 \\
D13 & 8.30 & 7 & D41 & 11.50 & 9 \\
D14 & 8.33 & 7 & D42 & 11.17 & 9 \\
D15 & 8.33 & 7 & D43 & 11.10 & 9 \\
D16 & 8.40 & 7 & D44 & 11.20 & 9 \\
D17 & 8.50 & 7 & D45 & 12.08 & 10 \\
D18 & 8.60 & 7 & D46 & 12.17 & 10 \\
D19 & 8.67 & 7 & D47 & 12.10 & 10 \\
D20 & 9.17 & 7 & D48 & 13.42 & 11 \\
D21 & 9.17 & 7 & D49 & 13.00 & 11 \\
D22 & 9.40 & 7 & D50 & 13.75 & 11 \\
D23 & 9.40 & 7 & D51 & 13.58 & 11 \\
D24 & 9.42 & 7 & D52 & 13.00 & 11 \\
D25 & 9.42 & 7 & D53 & 13.90 & 11 \\
D26 & 9.75 & 7 & D54 & 14.33 & 12 \\
D27 & 9.83 & 8 & D55 & 14.67 & 12 \\
D28 & 9.90 & 7 & & & \\
\hline & & & & & \\
\hline
\end{tabular}

from the children's parents after they were given an explanation about the experimental procedure.

\section{Ophthalmologic and Orthoptic Evaluation}

All children had normal values for ophthalmologic and orthoptic examination (showed in Table 2). The corrected visual acuity was normal $(\geq 20 / 20)$ for all children. All children had normal binocular vision evaluated with the TNO random dot test. The near point of convergence (NPC) was normal for all children. Heterophoria (i.e., the latent deviation of one covered eye when the other is not covered) measured using the cover-uncover test at near distance $(30 \mathrm{~cm})$ was normal for all children. Fusional amplitudes of convergence and divergence were measured at near distance $(30 \mathrm{~cm})$ using a base-in and a base-out prism bar; dyslexic children showed poorer convergence and divergence capabilities than the two groups of non-dyslexic children.

\section{Procedure}

Stimulus was presented on a $22^{\prime \prime}$ PC screen with a resolution of $1920 \times 1080$ and a refresh rate of $60 \mathrm{~Hz}$. The child was seated in a chair in a dark room, with his/her head stabilized by a forehead and chin support. Viewing was binocular; the viewing distance was $60 \mathrm{~cm}$. Calibration was done at the beginning of the fixation task. During the calibration procedure, the children were asked to fixate a grid of 13 points (diameter $0.5^{\circ}$ ) mapping the screen. Each calibration point required a fixation of $250 \mathrm{~ms}$ to be validated. A polynomial function with five parameters was used to fit the calibration data and to determine the visual angles. Afterwards, the child was invited to fixate a white-filled circle subtending a visual angle of $0.50^{\circ}$ appearing in the center of the screen during $30 \mathrm{~s}$. Two fixation tasks were recorded for each child.

\section{Eye Movement Recording}

Eye movements were recorded binocularly; horizontal and vertical eye positions were recorded independently and simultaneously for each eye with the EyeBrain T2, an eyetracking device CE-approved for medical applications. Recording frequency for both eyes was set up to $300 \mathrm{~Hz}$.

\section{Data Processing}

Calibration factors for each eye were determined from the eye positions during the calibration procedure (see Bucci et al., 2012). MeyeAnalysis ${ }^{\circledR}$ software (provided with the eye tracker) was used to extract the number of saccades during the fixation task. This software automatically detects both the onset and the offset of each saccade by using a built-in saccade detection algorithm. All detected saccades were manually checked by the investigator and corrected/discarded if necessary. All saccades $\geq 2^{\circ}$ were counted given that it is well known that microsaccades are normally smaller than such amplitude (Krekelberg, 2011). For each child (dyslexic and non-dyslexic) we counted the number of saccades measured in the two fixation tasks. Also, in order to evaluate visual attention in children, the number of saccade was also recorded in the first and in the last $15 \mathrm{~s}$ of fixation recording.

\section{Statistical Analysis}

Data were analyzed using the linear regression models for the three groups of children separately (dyslexic and chronological and reading age-matched non-dyslexic); dependant variable was the number of saccades measured during fixation task and

TABLE 2 | Clinical characteristics of children tested.

\begin{tabular}{|c|c|c|c|c|c|}
\hline Children (years) & TNO (s of arc) & PPC (cm) & Phoria (pD) & Convergence (pD) & Divergence $(\mathrm{pD})$ \\
\hline Dyslexics (10.2 \pm 0.2$)$ & $60 \pm 10.4$ & $3.00 \pm 2.2$ & $-2.2 \pm 0.7$ & $26.70 \pm 1.0$ & $10.60 \pm 0.5$ \\
\hline Reading age-matched non-dyslexics $(8.34 \pm 0.20)$ & $60 \pm 9.5$ & $2.00 \pm 2.0$ & $-2.4 \pm 0.5$ & $34.00 \pm 1.2$ & $16.02 \pm 1.2$ \\
\hline Chronological age-matched non-dyslexics (10.5 \pm 0.3$)$ & $66 \pm 15.5$ & $2.18 \pm 2.9$ & $-2.5 \pm 1.5$ & $35.00 \pm 0.8$ & $14.60 \pm 0.3$ \\
\hline
\end{tabular}

Note: clinical characteristics of the dyslexic and non-dyslexic children examined. Mean values \pm standard error of: binocular vision (Stereoacuity test, TNO measured in seconds of arc); near point of convergence (NPC measured in cm); Heterophoria at near distance measured in prism diopters (negative values indicate exophoria); Vergence fusional amplitudes (convergence and divergence) at near distance measured in prism diopters. 

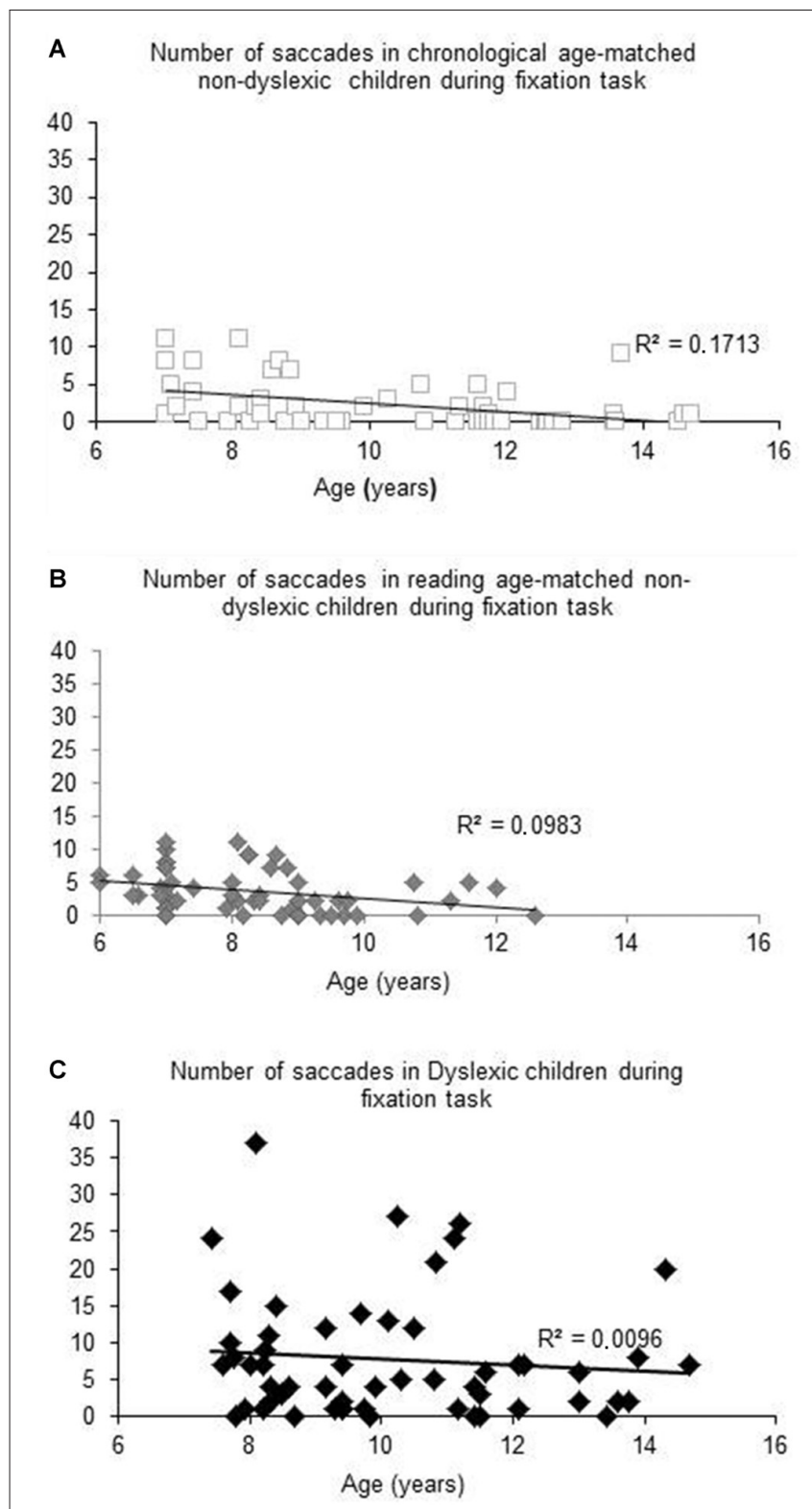

FIGURE 1 | Number of saccades measured during fixation task for Chronological age-matched non-dyslexic children (A), reading age-matched non-dyslexic children (B) and dyslexic children (C). Line represents the corresponding regression.

predictor variable was the children age (in years). An analysis of variance (ANOVA) was also performed with groups as intersubject factor and the number of saccades measured during fixation task (during all $30 \mathrm{~s}$ and also during the first and in the last $15 \mathrm{~s}$ of recording) as within-subject factors. Post hoc comparisons were made with the Fischer's test (LSD). The effect of a factor is significant when the $p$-value is below 0.05 .

\section{RESULTS}

Figure 1 shows the number of saccades measured during the total fixation task period as a function of the age (in years) of

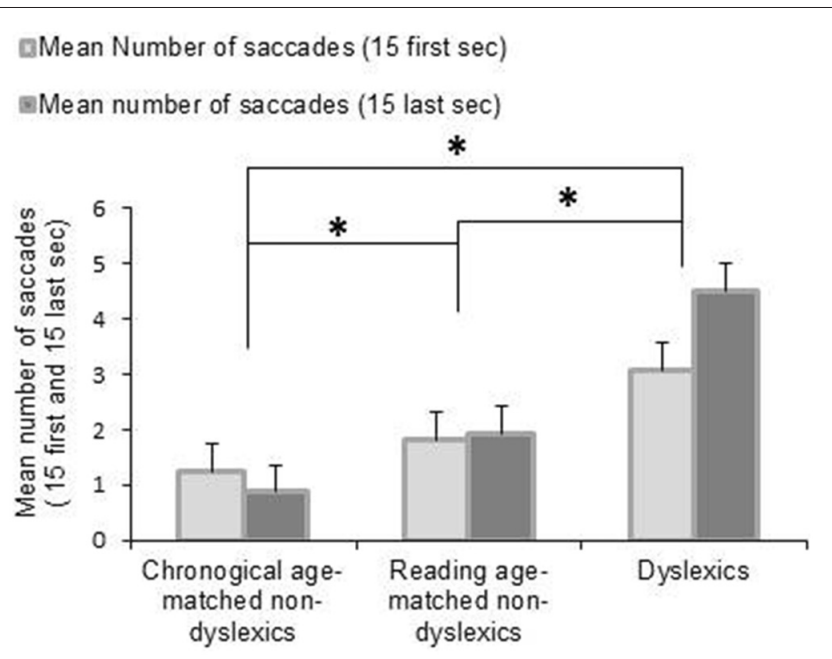

FIGURE 2 | Mean values of number of saccades during fixation task for the first 15 and the last $15 \mathrm{~s}$. Vertical bars indicate the standard error. Asterisk indicates a significant difference between the three groups of children.

each child tested and the regression line for both chronological (A) and reading age-matched non-dyslexic children (B) and dyslexic children (C). For both groups of non-dyslexic children, the number of saccades decreased significantly with the age of the children $\left(R^{2}=0.1727, p<0.02\right.$ and $R^{2}=0.0983$, $p<0.0001$, respectively for chronological and reading agematched non-dyslexic children). In contrast, for dyslexic children, the number of saccades did not decrease significantly with their chronological age $\left(R^{2}=0.0096, p=0.47\right)$ neither with their reading age $\left(R^{2}=0.031, p=0.19\right)$.

Figure 2 shows the mean number of saccades during the first 15 and the last $15 \mathrm{~s}$ of the fixation period for each group of children tested. The ANOVA revealed a main effect of group reflecting that the dyslexic readers generally made more saccades than the control groups $\left(F_{(2,162)}=14.999, p<0.0001\right)$. Moreover, we found an interaction between groups of children and period of fixation showing that dyslexic children made more saccades during the final half of the task than during the initial half $(p<0.0001)$-in the other two groups this was not the case. Post hoc test indicated also that dyslexic group made significant more number of saccades during the first $15 \mathrm{~s}$ of fixation with respect to the other two groups of non-dyslexic children (all $p<0.0001)$.

In order to know further the proportion of dyslexic children who made frequent saccades during fixation we showed in Table 3 the range and the different percentage of the number of saccades made by each group of children. Dyslexic children made the largest range of saccades (0-29 saccades) compared to the other two groups of children (0-11 saccades for chronological age-matched non-dyslexics group and 0-13 saccades for reading age-matched non-dyslexics group). Furthermore, the majority of the chronological age-matched control children (78\%) and $58 \%$ of reading age-matched children made three or less saccade in the fixation task. In contrast, about the half of dyslexic children (42\%) made three or less saccade in the 
TABLE 3 | Percentage of the number of saccades for each group tested.

\begin{tabular}{|c|c|c|c|}
\hline $\begin{array}{l}\text { Range of } \\
\text { saccades }\end{array}$ & $\begin{array}{l}\text { Chronological } \\
\text { age-matched } \\
\text { non-dyslexic } \\
\text { children (\%) }\end{array}$ & $\begin{array}{c}\text { Reading } \\
\text { age-matched } \\
\text { non-dyslexic } \\
\text { children (\%) }\end{array}$ & Dyslexics \\
\hline 0 & 49 & 24 & 15 \\
\hline 1 & 9 & 7 & 7 \\
\hline 2 & 15 & 16 & 11 \\
\hline 3 & 5 & 11 & 9 \\
\hline 4 & 4 & 5 & 9 \\
\hline 5 & 4 & 11 & 2 \\
\hline 6 & 0 & 2 & 4 \\
\hline 7 & 4 & 7 & 9 \\
\hline 8 & 4 & 4 & 4 \\
\hline 9 & 4 & 5 & 2 \\
\hline 10 & 0 & 2 & 2 \\
\hline 11 & 4 & 4 & 4 \\
\hline 12 & 0 & 0 & 2 \\
\hline 13 & 0 & 2 & 2 \\
\hline 14 & 0 & 0 & 5 \\
\hline 15 & 0 & 0 & 0 \\
\hline 16 & 0 & 0 & 0 \\
\hline 17 & 0 & 0 & 2 \\
\hline 18 & 0 & 0 & 0 \\
\hline 19 & 0 & 0 & 2 \\
\hline 20 & 0 & 0 & 0 \\
\hline 21 & 0 & 0 & 2 \\
\hline 22 & 0 & 0 & 0 \\
\hline 23 & 0 & 0 & 0 \\
\hline 24 & 0 & 0 & 2 \\
\hline 25 & 0 & 0 & 0 \\
\hline 26 & 0 & 0 & 2 \\
\hline 27 & 0 & 0 & 2 \\
\hline 28 & 0 & 0 & 0 \\
\hline 29 & 0 & 0 & 4 \\
\hline Total & 100 & 100 & 100 \\
\hline
\end{tabular}

Note: Range and different percentages of the number of saccades produced by each group of children tested.

fixation task while $58 \%$ of them made more than three saccades.

\section{DISCUSSION}

The main findings of this study are as follows: (i) the number of saccades during fixation is significantly higher in dyslexic children with respect to non-dyslexic children groups and (ii) the number of saccades during fixation significantly decreases with age in non-dyslexic children groups only. These findings are discussed below.

\section{The Number of Saccades During Fixation is Significantly Higher in Dyslexic with Respect to Non-Dyslexic Children}

Our data revealed that dyslexic children made a higher number of saccades during fixation than both non-dyslexic children groups. This study enlarges the previous work of Eden et al. (1994) in which poor fixation capability in 26 dyslexic children (11 years old) has been reported without counting the number of saccades during fixation task.
Our finding suggests that dyslexic children could have a weak ability to suppress unwanted saccades, most likely due to their difficulty in inhibition processing as well as to attentional deficits, even if we excluded in this study dyslexic with attentional deficiencies (see "Materials and Methods" Section). Such attentional impairment has been previously reported by Biscaldi et al. (1996), Facoetti et al. (2003) and de Araujo et al. (2015). More recently, Ruffino et al. (2010) studied attentional engagement in 28 dyslexics and 55 non-dyslexic children through a spatio-temporal attentional task with a fixation mark. Authors showed that dyslexic children only significantly exhibited an impaired identification of the targets and suggested that such impairment was linked to the attentional engagement deficit. Also, during single word reading, Thaler et al. (2009) reported that dyslexic children with attentional deficits made longer fixations than dyslexic without attentional deficits. In the present study, such attentional difficulty could be also substained by the fact that dyslexic children made significantly more saccades in the last 15 s during fixation task, while the two other groups made almost the same number of saccade in the first 15 and last $15 \mathrm{~s}$.

We also suggested that structures of the cortex responsible for saccade suppression and fixation control are immature in dyslexic children. Indeed, our data showed that reading agematched non-dyslexic children made smaller number of saccades than dyslexics suggesting that the reading experience does not explain the poor fixation reported in the present study in dyslexic children. These findings are in line with Clark et al.'s (2014) study concerning the neuroanatomical structures in dyslexics. These authors acquired reading and spelling data longitudinally via a RMI scans before the onset of reading learning and after dyslexia was diagnosed. They found that the visual area cortices were thinner in children who developed dyslexia. So, the cortical immaturity we suggested concerning the poor fixation in dyslexic children could be strictly linked with poor activity of SC and FEF neurons for a correct control of visual fixation. Several studies demonstrated the key role of SC and FEF in attention processing. In this line, Lovejoy and Krauzlis (2010) recorded the activity of SC in two monkeys after a muscimol injection and they reported a poor ability for them to focus their attention on a central fixation, suggesting that SC plays an important role in the selective attention. Krauzlis et al. (2013) also reported that SC is involved in spatial overt and covert attention. According to these authors, SC activity leads to spatial attention regulation during overt orienting eye movements. Basing their analysis on Ignashchenkova et al.'s (2004) study in which the shifts of attention in monkeys were analyzed, Krauzlis et al. (2013) also indicated the high activity of visual-motor SC neurons during covert shifts of attention. Thus, SC is important both in motor consequences as well as in visual-motor processing of attention. On the other hand, FEF neurons are known also to be involved in visual attention. Moore and Fallah (2001) simulated the FEF area in two monkeys while the latter performed a spatial attention task. The results showed that the enhancement of spatial attention is strongly related to the increase of FEF neurons activity and this has been confirmed in other studies on monkeys (Moore and Fallah, 2004) as well as on healthy subjects and patients with 
FEF lesions (Pierrot-Deseilligny et al., 2004; Esterman et al., 2015).

\section{The Number of Saccades During Fixation Significantly Decreases with Age in Non-Dyslexic Children Only}

This study showed that the number of saccades in both chronological and reading age-matched non-dyslexic groups significantly decreased with age, suggesting that the quality of the fixation improves with age. This finding is in line with previous studies exploring fixations in children populations (Munoz et al., 1998; Ajrezo et al., 2013) and showing that the quality of visual fixation in younger children is poor and improves until adolescence. Thus, the frontal and prefrontal cortices involved in fixation abilities as well as in saccade suppressions are still developing in young children. Other neurophysiological and neurocognitive studies (Sharpe and Zackon, 1987; Barkovich, 2000; Luna et al., 2004, 2008) showed that brain maturation is reached during adolescence (14 years old). Consequently we could make the hypothesis that the improvement of the visual fixation capabilities with age is related to a gradual and progressive maturation of such cortical structures until adolescence. On the other hand, the improvement of the quality of visual fixation with age could also be correlated with the maturation of visual attention capabilities in children. Indeed, it is well known that the attentional functions are immature in children. Konrad et al. (2005) investigated the neural mechanisms of attention in children from 8 to 12 years old using fRMI to assess the neural attentional networks. These networks involved right fronto-parietal regions for alerting, right temporo-parietal junction and right inferior frontal gyrus for orienting and reorienting, as well as anterior cingulate and lateral prefrontal cortex for executive attention. The results showed a weak activity of the attention regions that were assessed but an important activity of the superior areas and the occipital cortex. The authors concluded that the attentional functions are not mature but are in transition in children. These results could explain the weak inhibition capabilities in children from 8 to 12 years old, as was reported previously by Bunge et al. (2002).

\section{REFERENCES}

Ajrezo, L., Wiener-Vacher, S., and Bucci, M. P. (2013). Saccades improve postural control: a developmental study in normal children. PLoS One 8:e81066. doi: 10. 1371/journal.pone.0081066

Barkovich, A. J. (ed.). (2000). "Normal development of the neonatal and infant brain, skull and spine," in Pediatric Neuroimaging, (Philadelphia PA: Lippincott Williams and Wilkins), 13-69.

Biscaldi, M., Fischer, B., and Stuhr, V. (1996). Human express saccade makers are impaired at suppressing visually evoked saccades. J. Neurophysiol. 76, 199-214.

Braun, D., Weber, H., Mergner, T., and Schulte-Mönthing, J. (1992). Saccadic reaction time in patients with frontal and parietal lesions. Brain 115, 1359-1386. doi: 10.1093/brain/115.5.1359

Bucci, M. P., Bui-Quoc, E., and Gerard, C.-L. (2013a). The effect of a Strooplike task on postural control in dyslexic children. PLoS One 8:e77920. doi: 10. 1371/journal.pone.0077920
Based on all these findings, we suggested that the immaturity of these cortical areas in dyslexic children could lead to difficulties for them to focus their attention on the central target, leading to poor visual fixation performances compared with non-dyslexic age-matched children.

Finally, such poor fixation performance in dyslexic children could have a negative effect during reading given that during fixation period children extract lexical information from the words. So, such visual information processing could be responsible for longer fixations during reading, as reported by Bucci et al. (2012).

\section{CONCLUSION}

Our findings showed that dyslexic children exhibited many difficulties fixating a visual target and therefore triggered significantly more unwanted saccades than age-matched non-dyslexic children. An immaturity of cortical areas is most likely responsible for such poor fixation capabilities in dyslexic children. Orthoptic as well as visuo-attentional training in dyslexic children could help them to better focus their attention and therefore decrease reading errors and/or word omission.

\section{AUTHOR CONTRIBUTIONS}

AT: Performed the experiments, analyzed the data, wrote the article. C-LG: Contributed rearangements/ materials/analysis tools, wrote the article. HP: Contributed rearangements/materials/analysis tools, wrote the article. EB-Q: Contributed rearangements/materials/analysis tools, wrote the article. MPB: Conceived and designed the experiments, performed the experiments, wrote the article.

\section{ACKNOWLEDGMENTS}

The authors thank parents and children for their kind participation; Ms. Sandrine Larger for screening dyslexic children, and Paris Descartes Language Center for revising the English version of the manuscript.

Bucci, M. P., Gerard, C.-L., and Bui-Quoc, E. (2013b). The effect of a cognitive task on the postural control in dyslexic children. Res. Dev. Disabil. 34, 3727-3735. doi: 10.1016/j.ridd.2013.07.032

Bucci, M. P., Mélithe, D., Ajrezo, L., Bui-Quoc, E., and Gerard, C.-L. (2014). The influence of oculomotor tasks on postural control in dyslexic children. Front. Hum. Neurosci. 8:981. doi: 10.3389/fnhum.2014.00981

Bucci, M. P., Nassibi, N., Gerard, C.-L., Bui-Quoc, E., and Seassau, M. (2012). Immaturity of binocular saccade coordination in dyslexic children: evidence from a reading and visual search study. PLoS One 7:e33458. doi: 10. 1371/journal.pone.0033458

Bunge, S. A., Dudukovic, N. M., Thomason, M. E., Vaidya, C. J., and Gabrieli, J. D. (2002). Immature frontal lobe contributions to cognitive control in children: evidence from fMRI. Neuron 33, 301-311. doi: 10.1016/s0896-6273(01) 00583-9

Burman, D. D., and Bruce, C. J. (1997). Stimulation in the primate's frontal eye field suppression of task-related saccades by electrical. J. Neurophysiol. 77, 2252-2267. 
Chevrie-Muller, E. C., Simon, A. M., and Fournier, S. (1997). Batterie Langage Oral, Langage écrit, Mémoire, Attention (L2MA). Paris: Editions du Centre de Psychologie Appliquée.

Clark, K. A., Helland, T., Specht, K., Narr, K. L., Manis, F. R., Toga, A. W., et al. (2014). Neuroanatomical precursors of dyslexia identified from prereading through to age 11 . Brain 137, 3136-3141. doi: 10.1093/brain/ awu229

de Araujo, M. F. P., Matsumoto, J., Ono, T., and Nishijo, H. (2015). An animal model of disengagement: temporary inactivation of the superior colliculus impairs attention disengagement in rats. Behav. Brain Res. 293, 34-40. doi: 10. 1016/j.bbr.2015.07.031

De Luca, M., Di Pace, E., Judica, A., Spinelli, D., and Zoccoloti, P. (1999). Eye movement patterns in linguistic and non-linguistic tasks in developmental surface dyslexia. Neuropsychologia 37, 1407-1420. doi: 10. 1016/s0028-3932(99)00038-x

Eden, G. F., Stein, J. F., Wood, H. M., and Wood, F. B. (1994). Differences in eye movements and reading problems in dyslexic and normal children. Vision Res. 34, 1345-1358. doi: 10.1016/0042-6989(94)90209-7

Esterman, M., Liu, G., Okabe, H., Reagan, A., Thai, M., and DeGutis, J. (2015). Frontal eye field involvement in sustaining visual attention: evidence from transcranial magnetic stimulation. Neuroimage 111, 542-548. doi: 10.1016/j. neuroimage.2015.01.044

Facoetti, A., Lorusso, M. L., Paganoni, P., Cattaneo, C., Galli, R., and Mascetti, G. G. (2003). The time course of attentional focusing in dyslexic and normally reading children. Brain Cogn. 53, 181-184. doi: 10.1016/s0278-2626(03) 00105-2

Guitton, D., Buchtel, H. A., and Douglas, R. M. (1985). Frontal lesions in man cause difficulties in suppressing reflexive glances and in generating goal-directed saccades. Exp. Brain Res. 58, 455-472. doi: 10.1007/bf002 35863

Hasegawa, R. P., Peterson, B. W., and Goldberg, M. E. (2004). Prefrontal neurons coding suppression of specific saccades. Neuron 43, 415-425. doi: 10.1016/j. neuron.2004.07.013

Ignashchenkova, A., Dicke, P. W., Haarmeier, T., and Thier, P. (2004). Neuronspecific contribution of the superior colliculus to overt and covert shifts of attention. Nat. Neurosci. 7, 56-64. doi: 10.1038/nn1169

Konrad, K., Neufang, S., Thiel, C. M., Specht, K., Hanisch, C., Fan, J., et al. (2005). Development of attentional networks: an fMRI study with children and adults. Neuroimage 28, 429-439. doi: 10.1016/j.neuroimage.2005. 06.065

Krauzlis, R. J., Lovejoy, P., and Zénon, A. (2013). Superior colliculus and visual spatial attention. Ann. Rev. Neurosci. 36, 165-182. doi: 10.1146/annurev-neuro062012-170249

Krekelberg, B. (2011). Microsaccades. Curr. Biol. 21:R416. doi: 10.1016/j.cub.2011. 03.037

Leigh, R. J., and Zee, D. S. (2006). The Neurology of Eye Movement, 4th Ed. New York, NY: Oxford University Press.

Lovejoy, P., and Krauzlis, R. J. (2010). Inactivation of primate superior colliculus impairs covert selection of signals for perceptual judgments. Nat. Neurosci. 13, 261-266. doi: 10.1038/nn.2470

Luna, B., Garver, K. E., Urban, T. A., Lazar, N. A., and Sweeney, J. A. (2004). Maturation of cognitive processes from late childhood to adulthood. Child Dev. 75, 1357-1372. doi: 10.1111/j.1467-8624.2004.00745.x
Luna, B., and Sweeney, J. A. (2001). Studies of brain and cognitive maturation through childhood and adolescence: a strategy for testing neurodevelopmental hypotheses. Schizophr. Bull. 27, 443-455. doi: 10.1093/oxfordjournals.schbul. a006886

Luna, B., Velanova, K., and Geier, C. F. (2008). Development of eye-movement control. Brain Cogn. 68, 293-308. doi: 10.1016/j.bandc.2008.08.019

Moore, T., and Fallah, M. (2001). Control of eye movements and control of attention. Proc. Natl. Acad. Sci. U S A 98, 1273-1276. doi: 10.1073/pnas.98.3. 1273

Moore, T., and Fallah, M. (2004). Microstimulation of the frontal eye field and its effects on covert spatial attention. J. Neurophysiol. 91, 152-162. doi: 10.1152/jn. 00741.2002

Munoz, D. P., Broughton, J. R., Goldring, J. E., and Amstrong, I. T. (1998). Agerelated performance of human subjects on saccadic eye movement tasks. Exp. Brain Res. 121, 391-400. doi: 10.1007/s002210050473

Munoz, D. P., and Wurtz, R. H. (1992). Role of the rostral superior colliculus in active visual fixation and execution of express saccades. J. Neurophysiol. 67, 1000-10003.

Munoz, D. P., and Wurtz, R. H. (1995). Saccade-related activity in monkey superior colliculus. I. Characteristics of burst and buildup cells. J. Neurophysiol. 73, 2313-2333.

Palvidis, G. T. (1981). Do eye movements hold the key to dyslexia? Neuropsychologia 19, 57-64. doi: 10.1016/0028-3932(81)90044-0

Pierrot-Deseilligny, C., Milea, D., and Müri, R. M. (2004). Eye movement control by the cerebral cortex. Curr. Opin. Neurol. 17, 17-25. doi: 10.1097/00019052200402000-00005

Ruffino, M., Trussardi, A. N., Gori, S., Finzi, A., Giovagnolie, S., Menghini, D., et al. (2010). Attentional engagement deficits in dyslexic children. Neuropsychologia 48, 3793-3801. doi: 10.1016/j.neuropsychologia.2010.09.002

Segraves, M. A., and Park, K. (1993). The relationship of monkey frontal eye field activity to saccade dynamics. J. Neurophysiol. 69, 1880-1889.

Sharpe, J. A., and Zackon, D. H. (1987). Senescent saccades. Effects of aging on their accuracy, latency and velocity. Acta Otolaryngol. 104, 422-428. doi: 10. 3109/00016488709128270

Thaler, V., Urton, K., Heine, A., Hawelka, S., Engl, V., and Jacobs, A. M. (2009). Different behavioral and eye movement patterns of dyslexic readers with and without attentional deficits during single word reading. Neuropsychologia 47, 2436-2445. doi: 10.1016/j.neuropsychologia.2009.04.006

Tiadi, A., Seassau, M., Bui-Qoc, E., Gerard, C.-L., and Bucci, M. P. (2014). Vertical saccades in dyslexic children. Res. Dev. Disabil. 35, 3175-3181. doi: 10.1016/j. ridd.2014.07.057

Conflict of Interest Statement: The authors declare that the research was conducted in the absence of any commercial or financial relationships that could be construed as a potential conflict of interest.

Copyright (C) 2016 Tiadi, Gérard, Peyre, Bui-Quoc and Bucci. This is an open-access article distributed under the terms of the Creative Commons Attribution License (CC BY). The use, distribution and reproduction in other forums is permitted, provided the original author(s) or licensor are credited and that the original publication in this journal is cited, in accordance with accepted academic practice. No use, distribution or reproduction is permitted which does not comply with these terms. 